\title{
PREGRADO OPORTUNIDAD PARA APRENDER A INVESTIGAR
}

\author{
UNDERGRADUATE OPPORTUNITY TO LEARNTO RESEARCH
}

\author{
Naddyr Nataly Ojalvo Rojas ${ }^{1,2,4}$,Alejandra Sthefany Álvarez Alcocer ${ }^{1,2,3}$
}

$\mathrm{L}_{\mathrm{s}}^{\mathrm{a}}$ a formación científica en estudiantes de pregrado es muy importante, ya que proporciona un entrenamiento a dicha formación; por lo que el estudiante que incursiona en la investigación desarrolla aptitudes y cualidades diferentes a quienes no investigan, tales como el pensamiento crítico, la capacidad para indagar o ser curioso al buscar soluciones objetivas frente a situaciones específicas y el interés por la constante actualización de conocimientos, por ello el estudiante podrá plasmar sus ideas e innovarlas, asi como aportar algo novedoso a la comunidad científica por lo que esta formación en pregrado es útil para formar profesionales íntegros y con capacidades investigativas'.

En este sentido podemos decir que la investigación en pre-grado puede ser de vital importancia en la creación de futuros profesionales, quienes tengan la capacidad de afrontar las diferentes problemáticas en el país. Sin embargo, la investigación como tal es insuficiente, estas toman mayor relevancia cuando los resultados son publicados en revistas científicas y adquieren aun mayor impacto cuando es publicada en una revista indexada2.

Entre algunos factores que limitan la investigación en pregrado podemos mencionar: el escaso incentivo al estudiante por parte de la casa súper de estudios, la ausencia de asesoramiento y la poca o nula capacitación en los temas básicos de investigación como ser: metodología de la investigación, redacción de artículos científicos y proceso editorial de revistas ${ }^{2}$.

El entusiasmo del estudiante por incursionar en el mundo de la investigación se hace evidente con mayor fuerza al inicio de su formación académica con proyectos ambiciosos e ideas innovadoras, pero este ánimo se va perdiendo con el paso del tiempo; ya que de los trabajos que concluyen, una gran mayoría son presentados como requisito final en alguna asignatura (para luego ser archivados) o caso contrario no concluyen dicho trabajo por falta de tiempo y lo terminan solo por ser presentado a la materia, por lo que la motivación para terminarla se hace aún más ausente.

Sin embargo, para incentivar y potenciar el ámbito científico, los estudiantes de medicina formaron las llamadas sociedades científicas
(SoCEMs) que tiene como objetivo principal desarrollar las habilidades técnicasy teóricas en cuanto a investigación se refiere, por medio de la organización de charlas, talleres, cursos, jornadas y concursos entre otras actividades que se enfocan en temas como ser: metodología de la investigación, redacción de artículos científicos, ética en investigación, búsqueda bibliográfica entre otros ${ }^{3,4}$.

Estas sociedades científicas, ya sean locales o nacionales, se encuentran a nivel Latinoamérica agrupadas y conforman la Federación Latinoamericana de Sociedades Científicas de Estudiantes de Medicina (FELSOCEM), que tiene por objetivo "implementar los mecanismos para promover la inclusión científica en el área de las ciencias de la salud, a través de la educación médica continua, extensión universitaria y la formación médica integral en el pregrado". Hacía ya varios años la FELSOCEM coordina y organiza anualmente actividades de índole académico y científico (congresos nacionales e internacionales, concursos científicos, conferencias, campamentos, etc.) contribuyendo de esta manera a la formación científica de los estudiantes 
en pregrado, así como también aportando competencias que no son incluidas en las mallas curriculares universitarias. Ser miembro activo de una sociedad científica, no solo implica cumplir con los requisitos mínimos como ser el pago de la membresía, sino también el participar activamente de las actividades desarrolladas por la misma, por ejemplo realizar investigación, publicarla y así generar nuevos conocimientos que pueden ser llevados a un congreso científico aumentado asi el prestigio de la sociedad científica, lo que contribuirá al aprendizaje del estudiante en el ámbito científico, el ser parte de estas sociedades científicas tambien tiene como beneficio la capacitación continua ya sea por parte de la misma directiva o doctores invitados especializados en el área, que serán quienes darán un enfoque más práctico y didáctico a quienes inician su camino a la investigacion ${ }^{3,4}$.

Muchos autores coinciden en que gracias a las sociedades científicas, los estudiantes adquieren mayor y mejor conocimiento al potenciar sus aptitudes en el ámbito investigativo, así como también contribuye a su formación como futuros médicos e investigadores, mediante la realización de sus trabajos de investigación y finalizando el mismo al ser publicados en revistas indexadas.

Por lo tanto llegamos a la conclusión de que a pesar de las limitantes y obstáculos que los estudiantes tienen en el camino a la investigación, muchos se dan maneras de aprender $y$ evitan asi dejar esa curiosidad de investigadores de lado, al formar parte de dichas sociedades científicas quienes brindan la oportunidad para desarrollar la investigación estudiantil y el aprendizaje médico-científico, por lo que aportan los pilares fundamentales y los cimientos necesarios para que el estudiante arranque como futuro investigador y como futuro medico quien tendrá esa vocación y la capacidad de plasmar sus ideas, innovarlas y finalmente publicarlas como un nuevo descubrimiento el cual pueden ser la solución a muchos problemas en salud 5 .

Palabras clave: aprendizaje científico, sociedades científicas, educación médica.

Keywords: scientific learning, scientific societies, medical education.

\section{1,2,4 Naddyr Nataly Ojalvo Rojas}

\section{1,2,3 Alejandra Sthefany Álvarez Alcocer} 1. Editor asociado de la Revista Cientifica

Ciencia Médica

2. Sociedad Cientifica de Estudiantes de Medicina

3. Facultad de Medicina "Aurelio Melean", Universidad Mayor de San Simón. Cochabamba-Bolivia.

4. Facultad de Medicina, Universidad Privada Franz Tamayo. Cochabamba-Bolivia

Correspondencia a: Naddyr Nataly Ojalvo Rojas

Correo electronico: naddyr14@gmail. com

\section{REFERENCIAS}

1.Tapia AEC. Investigación y la formación científica en pregrado de Medicina. Discov Med [Internet]. 2017 Jul 3 [citado 2021 Ene 25];1(1):57-60. Disponible en: https://www. revdiscovermedicine.com/index.php/inicio/article/ view/14

2.Vargas-Mamani J, Investigación en pregrado 20 años de revista Científica "Ciencia Médica". Rev Cient Cien Med[Internet].2017 [citado 2021 Ene 28]; 20(2): 3-4 Disponible en: http://www.scielo. org.6o/scielo. php? script $=$ sci_issuetoceLpid $=1817$ -

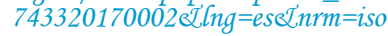

3.Aveiro-Róbalo TR, Escobar-Salinas JS, Ayala-Servín JN, Rotela-Fisch V. Importancia de las sociedades científicas de estudiantes de medicina en Latinoamérica. Investigación en educación médica [Internet]. [citado 2021 Ene 14];8(29):23-9. Disponible en: https://www.redalyc.org/ jatsRepo/3497/349760784004/html/index.html

4.¿Cómo impulsar una sociedad científica de estudiantes de medicina? Educación Médica [Internet]. 2019 Mar 1 [cited 2021 Ene 14];20:175-85. Disponible en: http:// dx.doi.org/10.1016/j.edumed.2017.11.009

5.Ortiz-Martínez Y.ROLDE LAS SOCIEDADES CIENTIFICAS EN LA INVESTIGACION MEDICA ESTUDIANTIL EN COLOMBIA. Rev Cient Cienc Méd [Internet]. 2016 [citado 2021 Ene 25];19(1):66-7. Disponible en: http://www.scielo.org. 6o/scielo.php? script $=$ sci

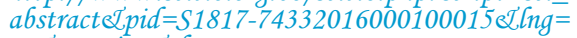

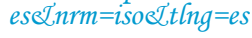

Citar como:

Ojalvo Rojas NN, Álvarez Alcocer AS. Pregrado oportunidad para aprender a investigar. Rev Cient Cienc Med. 2020; 23(2). 